\title{
Isospin breaking in pion Compton scattering
}

\author{
N. Kaiser ${ }^{\mathrm{a}}$ \\ Physik-Department, Technische Universität München, D-85747 Garching, Germany
}

Received: 28 October 2010 / Revised: 9 December 2010

Published online: 1 February 2011

(c) The Author(s) 2011. This article is published with open access at Springerlink.com

Communicated by J. Bijnens

\begin{abstract}
Using chiral perturbation theory we calculate for pion Compton scattering the isospin-breaking effects induced by the difference between the charged and neutral pion mass. At one-loop order this correction is directly proportional to $m_{\pi^{ \pm}}^{2}-m_{\pi^{0}}^{2}$ and free of (electromagnetic) counterterm contributions. The differential cross-section for charged pion Compton scattering $\pi^{-} \gamma \rightarrow \pi^{-} \gamma$ gets affected (in backward directions) at the level of a few permille. At the same time the isospin-breaking correction leads to a small shift of the pion polarizabilities by $\delta\left(\alpha_{\pi}-\beta_{\pi}\right) \simeq 1.3 \cdot 10^{-5} \mathrm{fm}^{3}$. In case of the low-energy $\gamma \gamma \rightarrow \pi^{0} \pi^{0}$ reaction isospin breaking manifests itself through a cusp effect at the $\pi^{+} \pi^{-}$threshold. We give an improved estimate for it based on the empirical $\pi \pi$-scattering length difference $a_{0}-a_{2}$.
\end{abstract}

\section{Introduction and summary}

At present there is much interest in a precise experimental determination of the pion electric and magnetic polarizabilities, $\alpha_{\pi}$ and $\beta_{\pi}$. Within the framework of current algebra [1] it has been shown (long ago) that the polarizability difference $\alpha_{\pi}-\beta_{\pi}$ of the charged pion is directly related to the axial-vector-to-vector form factor ratio $h_{A} / h_{V} \simeq 0.44$ measured in the radiative pion decay $\pi^{+} \rightarrow e^{+} \nu_{e} \gamma[2]$. At leading (non-trivial) order the result of chiral perturbation theory [3], $\alpha_{\pi}-\beta_{\pi}=\alpha\left(\bar{l}_{6}-\bar{l}_{5}\right) /\left(24 \pi^{2} f_{\pi}^{2} m_{\pi}\right)$, is of course the same after identifying the low-energy constant as $\bar{l}_{6}-\bar{l}_{5}=6 h_{A} / h_{V}$. Recently, the systematic corrections to this current algebra result have been worked out in refs. $[4,5]$ by performing a full two-loop calculation of pion Compton scattering in chiral perturbation theory. The outcome of that extensive analysis is that altogether the higher-order corrections are rather small and the value $\alpha_{\pi}-\beta_{\pi}=(5.7 \pm 1.0) \cdot 10^{-4} \mathrm{fm}^{3}[5]$ stands now as a firm prediction of the (chiral-invariant) theory. It is however in conflict with the existing experimental determinations of $\alpha_{\pi}-\beta_{\pi}=(15.6 \pm 7.8) \cdot 10^{-4} \mathrm{fm}^{3}$ from Serpukhov [6] and $\alpha_{\pi}-\beta_{\pi}=(11.6 \pm 3.4) \cdot 10^{-4} \mathrm{fm}^{3}$ from Mainz [7], which amount to values more than twice as large. Certainly, these existing experimental determinations of $\alpha_{\pi}-\beta_{\pi}$ raise doubts about their correctness since they violate the chiral low-energy theorem notably by a factor 2 . In this context it is worth to note that a recent dispersive analysis [8] of the Belle and Crystal Ball data for $\gamma \gamma \rightarrow \pi^{+} \pi^{-}$ gives the fit value $\alpha_{\pi}-\beta_{\pi}=4.7 \cdot 10^{-4} \mathrm{fm}^{3}$, compatible with chiral perturbation theory. The non-vanishing

\footnotetext{
a e-mail: nkaiser@ph.tum.de
}

two-loop prediction for the polarizability sum, $\alpha_{\pi}+\beta_{\pi}=$ $(0.16 \pm 0.1) \cdot 10^{-4} \mathrm{fm}^{3}[5]$, is actually consistent with results from dispersion sum rules [9] but presumably too small to cause an observable effect in low-energy pion Compton scattering.

In that situation it is promising that the ongoing COMPASS experiment [10] at CERN aims at remeasuring the pion polarizabilities, $\alpha_{\pi}$ and $\beta_{\pi}$, with high statistics using the Primakoff effect. The scattering of high-energy negative pions in the Coulomb field of a heavy nucleus (of charge $Z$ ) gives access to crosssections for $\pi^{-} \gamma$ reactions through the equivalent photon method [11]. The consistent theoretical framework to extract the pion polarizabilities from the measured cross-sections for (low-energy) pion Compton scattering $\pi^{-} \gamma \rightarrow \pi^{-} \gamma$ or the primary pion-nucleus bremsstrahlung process $\pi^{-} Z \rightarrow \pi^{-} Z \gamma$ has been described (in one-loop approximation) in refs. $[12,13]$. It has been stressed that at the same order as the polarizability difference $\alpha_{\pi}-\beta_{\pi}$ there exists a further (partly compensating) pion structure effect in form of a unique pion-loop correction (interpretable as photon scattering off the "pion-cloud around the pion"). In addition to these strong interaction effects, the QED radiative corrections to real and virtual pion Compton scattering $\pi^{-} \gamma^{(*)} \rightarrow \pi^{-} \gamma$ have been calculated in refs. $[13,14]$. The relative smallness of the pion structure effects in low-energy pion Compton scattering [12] makes it necessary to include such higher-order electromagnetic corrections.

There exists yet another correction which is predominantly of electromagnetic origin, namely the isospinbreaking effect induced in pion loops via the charged and neutral pion mass difference. The purpose of the present 
letter is to work out this correction for pion Compton scattering in the systematic framework of chiral perturbation theory. We find that at one-loop order this isospinbreaking correction is directly proportional to $m_{\pi^{ \pm}}^{2}-m_{\pi^{0}}^{2}$ and free of unknown (electromagnetic) counterterm contributions. The differential cross-section for the charged pion Compton scattering $\pi^{-} \gamma \rightarrow \pi^{-} \gamma$ gets affected (in backward directions) at the level of a few permille only. At the same time the isospin-breaking correction leads to a small shift of the pion polarizability difference by $\delta\left(\alpha_{\pi}-\beta_{\pi}\right) \simeq 1.3 \cdot 10^{-5} \mathrm{fm}^{3}$. In case of the low-energy $\gamma \gamma \rightarrow \pi^{0} \pi^{0}$ reaction isospin breaking manifests itself through a cusp effect at the $\pi^{+} \pi^{-}$threshold. The occurrence of the cusp in the corresponding total cross-section is primarily of kinematical origin (i.e. it is made accessible by a phase space factor). We give an improved estimate for the cusp effect in $\gamma \gamma \rightarrow \pi^{0} \pi^{0}$ by employing the empirical $\pi \pi$-scattering length difference $a_{0}-a_{2} \simeq 0.26 m_{\pi}^{-1}$.

In comparison to the "genuine" radiative corrections (arising from virtual photon loops and soft photon bremsstrahlung) the induced isospin-breaking correction is suppressed by one order of magnitude. Therefore it contributes to pion Compton scattering well beyond the (expected) accuracy of present or near future experiments. One should note that similar isospin-breaking corrections have been studied in detail for the pionium decay amplitude $\left(\pi^{+} \pi^{-} \rightarrow \pi^{0} \pi^{0}\right)$ in ref. [15], reaching the conclusion that these can be as large as $3 \%$. From that perspective the extreme smallness of the (dynamical) isospin-breaking correction to pion Compton scattering represents a nontrivial result.

\section{Charged pion Compton scattering}

We start out with defining the invariant amplitudes for the pion Compton scattering process: $\pi^{-}\left(p_{1}\right)+\gamma\left(k_{1}, \epsilon_{1}\right) \rightarrow$ $\pi^{-}\left(p_{2}\right)+\gamma\left(k_{2}, \epsilon_{2}\right)$. The corresponding $T$-matrix in the center-of-mass frame has (in Coulomb gauge $\epsilon_{1,2}^{0}=0$ ) the form:

$T_{\pi \gamma}=8 \pi \alpha\left\{-\vec{\epsilon}_{1} \cdot \vec{\epsilon}_{2}^{*} A(s, t)+\vec{\epsilon}_{1} \cdot \vec{k}_{2} \vec{\epsilon}_{2}^{*} \cdot \vec{k}_{1} \frac{2}{t}[A(s, t)+B(s, t)]\right\}$,

with $\alpha=1 / 137.036$ the fine-structure constant, and $s=$ $\left(p_{1}+k_{1}\right)^{2}>m_{\pi}^{2}$ and $t=\left(k_{1}-k_{2}\right)^{2}<0$ the independent Mandelstam variables. $m_{\pi}=139.570 \mathrm{MeV}$ denotes the charged pion mass. Performing the sums over transversal photon polarizations and applying the flux and phase space factors, the resulting differential cross-section reads:

$$
\frac{\mathrm{d} \sigma}{\mathrm{d} \Omega_{\mathrm{cm}}}=\frac{\alpha^{2}}{2 s}\left\{|A(s, t)|^{2}+|A(s, t)+(1+z) B(s, t)|^{2}\right\},
$$

with $t=\left(s-m_{\pi}^{2}\right)^{2}(z-1) / 2 s$, where $z=\cos \theta_{\mathrm{cm}}=\hat{k}_{1} \cdot \hat{k}_{2}$ is the cosine of the cms scattering angle. The decomposition in eq. (1) into two invariant amplitudes $A(s, t)$ and $B(s, t)$ has been done with hindsight to an expression as simple as possible for the (unpolarized) differential cross-section. The amplitudes at tree level coincide with those of scalar
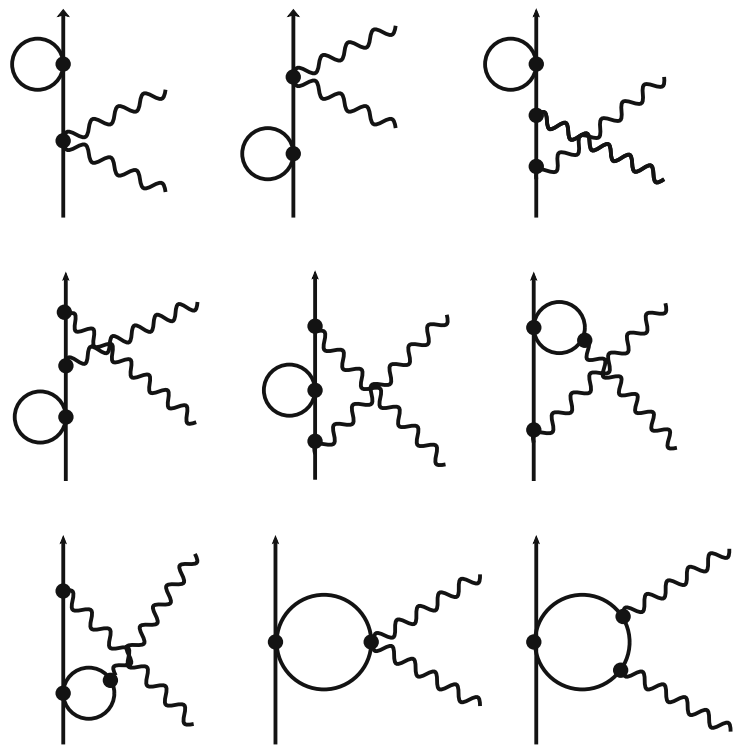

Fig. 1. Relevant set of pion-loop diagrams for the pion Compton scattering. Except for the last diagram the combinatorial factor is $1 / 2$.

quantum electrodynamics:

$$
A(s, t)^{(\text {tree })}=1, \quad B(s, t)^{(\text {tree })}=\frac{s-m_{\pi}^{2}}{m_{\pi}^{2}-s-t} .
$$

Loop corrections are generated by the vertices from the leading-order chiral Lagrangian:

$$
\mathcal{L}_{\pi \pi}^{(2)}=\frac{f_{\pi}^{2}}{4} \operatorname{tr}\left(D_{\mu} U D^{\mu} U^{\dagger}+m_{\pi^{0}}^{2}\left(U+U^{\dagger}\right)\right),
$$

and the corresponding one-pion loop diagrams are shown in fig. 1. Note that in Coulomb gauge all $s$-channel pole diagrams vanish, since $\epsilon_{1} \cdot\left(2 p_{1}+k_{1}\right)=0=\epsilon_{2} \cdot\left(2 p_{2}+\right.$ $\left.k_{2}\right)$. Furthermore, in the convenient parameterization of the special-unitary matrix field $U=\sqrt{1-\vec{\pi}^{2} / f_{\pi}^{2}}+i \vec{\tau}$. $\vec{\pi} / f_{\pi}$ no $\gamma 4 \pi$ and $2 \gamma 4 \pi$ contact-vertices exist at (leading order). After renormalization of the pion mass one obtains the following (finite) loop contribution to the Compton amplitudes $[4,5,12]$ :

$$
\begin{aligned}
& A(s, t)^{(\mathrm{loop})}=\frac{1}{\left(4 \pi f_{\pi}\right)^{2}}\left\{-\frac{t}{2}-2 m_{\pi}^{2} \ln ^{2} \frac{\sqrt{4 m_{\pi}^{2}-t}+\sqrt{-t}}{2 m_{\pi}}\right\}, \\
& B(s, t)^{(\mathrm{loop})}=0,
\end{aligned}
$$

where $f_{\pi}=92.4 \mathrm{MeV}$ denotes the pion decay constant. As written in eq. $(5), A(s, t)^{(\text {loop })}$ corresponds to the limit of (exact) isospin symmetry, in which the difference between the charged pion mass, $m_{\pi}=139.570 \mathrm{MeV}$, and the neutral pion mass, $m_{\pi^{0}}=134.977 \mathrm{MeV}$, is neglected. The isospin-breaking pion mass difference is mainly of electromagnetic origin and it can be accounted for systematically in chiral perturbation theory by the effective Lagrangian [16]:

$$
\mathcal{L}_{e^{2}}=C \operatorname{tr}\left(Q_{R} U Q_{L} U^{\dagger}\right),
$$


which is counted (together with $\mathcal{L}_{\pi \pi}^{(2)}$ ) as a leading-order one. By setting the auxiliary "spurion" fields equal to the (two-flavor) quark charge matrix $Q_{L}=Q_{R}=$ $\frac{e}{3} \operatorname{diag}(2,-1)$ one deduces the relation $m_{\pi}^{2}-m_{\pi^{0}}^{2}=$ $2 C e^{2} / f_{\pi}^{2}$ for the pion mass difference. Note that in the above-mentioned parameterization of the chiral field $U=$ $\sqrt{1-\vec{\pi}^{2} / f_{\pi}^{2}}+i \vec{\tau} \cdot \vec{\pi} / f_{\pi}$ the Lagrangian $\mathcal{L}_{e^{2}}$ provides only the mass splitting term for the charged pions but no further (isospin-breaking) pion-pion interaction terms. As a result of this feature the additional Lagrangian $\mathcal{L}_{e^{2}}$ introduces in the loop diagrams of fig. 1 for all propagators of charged pions their correct mass square $m_{\pi}^{2}>m_{\pi^{0}}^{2}$. The isospin-breaking correction to eq. (5) is therefore determined by the residual dependences of the Compton scattering diagrams on $m_{\pi^{0}}^{2}$ either through neutral pion propagators or through the chiral $4 \pi$-interaction vertex. The first five (self-energy-type) diagrams in fig. 1 introduce the wave function renormalization factor $Z_{2}^{( \pm)}-1$ of the charged pion. This quantity receives no contribution from neutral pion loops and it is determined solely by the momentum-dependent part of the chiral $4 \pi$ interaction (i.e. the one independent of $m_{\pi^{0}}^{2}$ ). The remaining four diagrams in fig. 1 involve obviously only charged pions. For the two reducible (vertex-type) diagrams again only the momentum-dependent part of the chiral $4 \pi$-interaction plays a role, since the contribution from the chiral symmetry-breaking mass term vanishes: $\epsilon_{c 3 d}\left(\delta_{a b} \delta_{c d}+\delta_{a c} \delta_{b d}+\delta_{a d} \delta_{b c}\right)=\epsilon_{a 3 b}+\epsilon_{b 3 a}=0$. The isospinbreaking correction arises therefore entirely from the $m_{\pi^{0}}^{2}$ dependence of the chiral $4 \pi$-interaction vertex entering the last two (irreducible) diagrams in fig. 1. After performing the pertinent loop calculation one finds the following analytical expression for the isospin-breaking correction:

$A(s, t)^{(\mathrm{isobr})}=\frac{m_{\pi}^{2}-m_{\pi^{0}}^{2}}{\left(2 \pi f_{\pi}\right)^{2}}\left\{-\frac{1}{2}-\frac{2 m_{\pi}^{2}}{t} \ln ^{2} \frac{\sqrt{4 m_{\pi}^{2}-t}+\sqrt{-t}}{2 m_{\pi}}\right\}$,

$B(s, t)^{(\text {isobr })}=0$,

in which the ultraviolet divergences from individual diagrams have canceled. Since the low-energy expansion of $A(s, t)^{\text {(isobr) }}$ starts with a linear term in $t$, it gives rise to a contribution to the pion polarizability difference (see, e.g., eq. (6) in ref. [12]):

$$
\delta\left(\alpha_{\pi}-\beta_{\pi}\right)=\frac{\alpha\left(m_{\pi}^{2}-m_{\pi^{0}}^{2}\right)}{24 \pi^{2} f_{\pi}^{2} m_{\pi}^{3}} \simeq 1.3 \cdot 10^{-5} \mathrm{fm}^{3} .
$$

This small shift amounts to about $2.3 \%$ of the chiral twoloop prediction $\alpha_{\pi}-\beta_{\pi}=(5.7 \pm 1.0) \cdot 10^{-4} \mathrm{fm}^{3}$ of ref. [5] based on isospin symmetry and it is well covered by the estimated theoretical errorbar. Figure 2 shows more directly the effect of the isospin-breaking correction $A(s, t)^{\text {(isobr) }}$ on the differential cross-section for pion Compton scattering $\pi^{-} \gamma \rightarrow \pi^{-} \gamma$. The four curves correspond to centerof-mass energies $\sqrt{s}=(2,3,4,5) m_{\pi}$ and each gives, as a function of $z=\cos \theta_{\mathrm{cm}}$, the change of the differential crosssection caused by the amplitude $A(s, t)^{\text {(isobr) }}$ in proportion to the tree level cross-section. One observes negative corrections of merely a few permille which become maximal

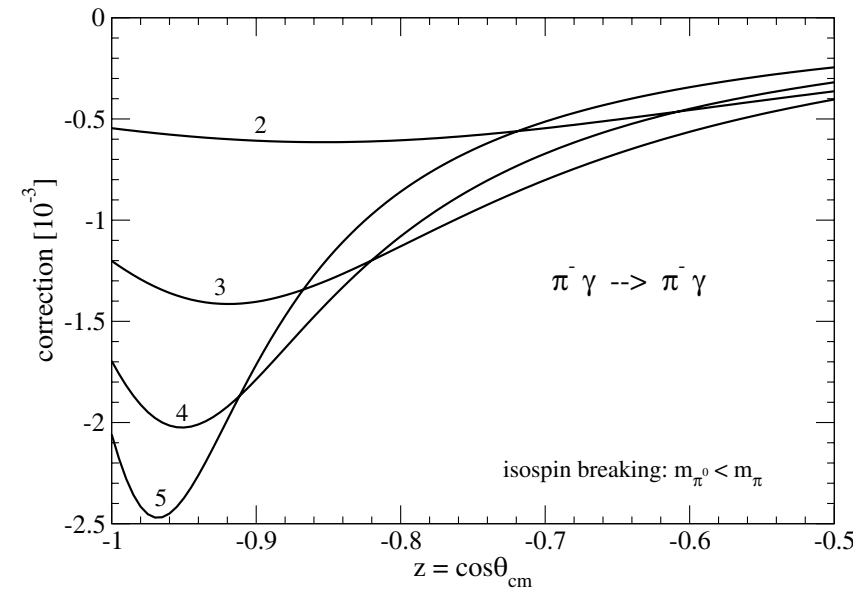

Fig. 2. Isospin-breaking correction to charged pion Compton scattering. The numbers $2,3,4,5$ on the curves correspond to the center-of-mass energy $\sqrt{s}$ divided by $m_{\pi}$.

in backward directions $(z \simeq-1)$. The isospin-breaking correction has therefore a similar kinematical signature as the "genuine" radiative corrections (arising from virtual photon loops and soft photon bremsstrahlung) but it is suppressed by one order of magnitude (see fig. 3 in ref. [13]).

Although the isospin-breaking loop amplitude $A(s, t)^{(\text {isobr) }}$ in eq. (7) is finite there could eventually exist additional contributions at order $\mathcal{O}\left(e^{2} p^{2}\right)$ from local counterterms. The complete list of these counterterms has been given in eq. (3.6) of ref. [16] and for the present application they all reduce to the form: $k_{j}\left(\partial_{\mu} \phi^{\dagger}-i e A_{\mu} \phi^{\dagger}\right)\left(\partial^{\mu} \phi+i e A^{\mu} \phi\right)$. Since they are proportional to the gauged Klein-Gordon Lagrangian for charged pions they can simply be eliminated by a redefinition of the pion field. Alternatively, one can show that the contributions arising in the form of pion-photon vertex corrections and $Z_{2}$-factor insertions cancel each other exactly. A further argument for the absence of any counterterm contributions to pion Compton scattering at order $\mathcal{O}\left(e^{2} p^{2}\right)$ is provided by the non-renormalization theorem for the Thomson amplitude: $\lim _{k_{1,2} \rightarrow 0} T_{\pi \gamma}=-8 \pi \alpha \vec{\epsilon}_{1} \cdot \vec{\epsilon}_{2}^{*}$.

\section{Neutral pion Compton scattering}

For the sake of completeness we consider also the isospinbreaking correction to neutral pion Compton scattering $\pi^{0} \gamma \rightarrow \pi^{0} \gamma$. In that case the tree amplitudes vanish and the leading contribution arises from the last two (irreducible) pion-loop diagrams in fig. 1. Taking into account the different masses of charged and neutral pions the resulting loop amplitudes read:

$$
\begin{aligned}
& A_{n}(t)=\frac{t-m_{\pi^{0}}^{2}}{\left(4 \pi f_{\pi}\right)^{2}}\left\{-1-\frac{4 m_{\pi}^{2}}{t} \ln ^{2} \frac{\sqrt{4 m_{\pi}^{2}-t}+\sqrt{-t}}{2 m_{\pi}}\right\}, \\
& B_{n}(t)=0 .
\end{aligned}
$$

Note that the prefactor $\left(t-m_{\pi^{0}}^{2}\right) / f_{\pi}^{2}$ originates from the chiral $\pi^{+} \pi^{-} \rightarrow \pi^{0} \pi^{0}$ transition amplitude (at leading or- 


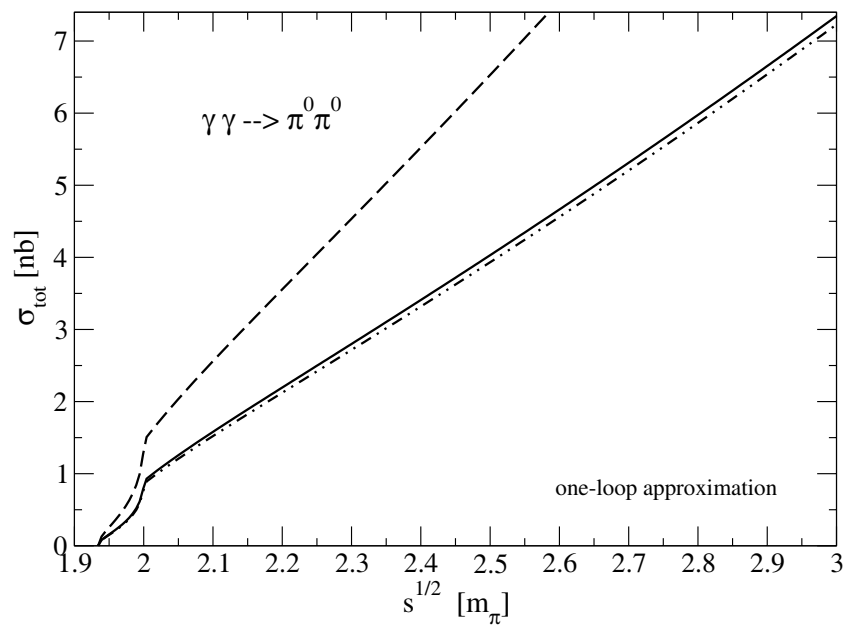

Fig. 3. Total cross-section for the reaction $\gamma \gamma \rightarrow \pi^{0} \pi^{0}$.

der). The Compton amplitude $A_{n}(t)$ determines at the same time the total cross-section for the (crossed) reaction $\gamma \gamma \rightarrow \pi^{0} \pi^{0}$ as $[17,18]$ :

$$
\sigma_{\text {tot }}\left(\gamma \gamma \rightarrow \pi^{0} \pi^{0} ; s\right)=\frac{\pi \alpha^{2}}{s^{3 / 2}} \sqrt{s-4 m_{\pi^{0}}^{2}}\left|A_{n}(s)\right|^{2},
$$

with $\sqrt{s}$ the corresponding center-of-mass energy. The proper analytical continuation of the squared logarithm in eq. (9) is $[\ln (\sqrt{x-1}+\sqrt{x})-i \pi / 2]^{2}$ for $x=s / 4 m_{\pi}^{2}>1$, and $-\arcsin ^{2} \sqrt{x}$ for $0<x<1$. The full line in fig. 3 shows the total cross-section $\sigma_{\text {tot }}\left(\gamma \gamma \rightarrow \pi^{0} \pi^{0} ; s\right)$ resulting in the one-loop approximation in the region near threshold $2 m_{\pi^{0}}<\sqrt{s}<3 m_{\pi}$. The novel feature of including isospin breaking is a cusp effect at the $\pi^{+} \pi^{-}$threshold. The occurrence of the cusp is primarily a kinematical effect, since it is the (isospin-breaking) two-body phase space factor $\sqrt{s-4 m_{\pi^{0}}^{2}}$ in eq. (10) that gives access to the production amplitude $A_{n}(s)$ below its branch point $s=4 m_{\pi}^{2}$. This feature is clearly demonstrated by the dash-dotted line in fig. 3 which follows after dropping the isospin-breaking pion mass difference $\left(m_{\pi^{0}} \rightarrow m_{\pi}\right)$ in the squared production amplitude $\left|A_{n}(s)\right|^{2}$. The actual strength of the cusp is governed by the $\pi^{+} \pi^{-} \rightarrow \pi^{0} \pi^{0}$ transition amplitude at threshold, or equivalently the difference between the isospin-zero and isospin-two $S$-wave $\pi \pi$-scattering lengths $a_{0}-a_{2}$. It is well known that this quantity increases by about $25 \%$ after inclusion of next-to-leading-order chiral corrections. Therefore the cusp effect will come out more pronounced in a two-loop calculation of the low-energy photon-photon collision process $\gamma \gamma \rightarrow \pi^{0} \pi^{0}$. For that purpose it is instructive to compare the one-loop and two-loop amplitudes displayed in fig. 8 of ref. [19] in the region around the $\pi^{+} \pi^{-}$threshold $s=4 m_{\pi}^{2}$, and extract from these curves the enhancement factor (about 1.6) for the cusp effect. The dashed line in fig. 3 shows the result for the near-threshold cross-section obtained with an improved treatment of the $\pi^{+} \pi^{-} \rightarrow \pi^{0} \pi^{0}$ interaction using the empirical $\pi \pi$-scattering length difference $a_{0}-a_{2}=0.26 m_{\pi}^{-1}[20]$ instead of the leading-order chiral result $a_{0}-a_{2}=9 m_{\pi} /\left(32 \pi f_{\pi}^{2}\right)=0.204 m_{\pi}^{-1}$. It is expected that such a simple (rescaling) approach is fairly accurate in the close vicinity of the $\pi^{+} \pi^{-}$threshold $s=4 m_{\pi}^{2}$. As a check a more detailed investigation of the cusp effect in the $\gamma \gamma \rightarrow \pi^{0} \pi^{0}$ process which implements exact elastic unitarity should be performed along the lines of the work by Cabibbo and Isidori [21]. Let us also note that the same type of cusp occurring in the related process $\pi^{0} \pi^{0} \rightarrow \pi^{0} \pi^{0}$ has been calculated some time ago in ref. [22]. While from the theoretical side the occurrence of a cusp in $\sigma_{\text {tot }}\left(\gamma \gamma \rightarrow \pi^{0} \pi^{0} ; s\right)$ is quite natural, it represents a very big challenge to reveal it also experimentally. The present status of the low-energy data for the reaction $\gamma \gamma \rightarrow \pi^{0} \pi^{0}$ is summarized in fig. 7 of ref. [8]. In the region from threshold up to $\sqrt{s}=3 m_{\pi}$ there exist presently only two data points with sizeable errorbars.

Open Access This article is distributed under the terms of the Creative Commons Attribution Noncommercial License which permits any noncommercial use, distribution, and reproduction in any medium, provided the original author(s) and source are credited.

\section{References}

1. M.V. Terentev, Sov. J. Nucl. Phys. 16, 87 (1973).

2. E. Frlez et al., Phys. Rev. Lett. 93, 181804 (2004).

3. J.F. Donoghue, B.R. Holstein, Phys. Rev. D 40, 2378 (1989).

4. U. Bürgi, Nucl. Phys. B 479, 392 (1996).

5. J. Gasser, M.A. Ivanov, M.E. Sainio, Nucl. Phys. B 745, $84(2006)$.

6. Y.M. Antipov et al., Z. Phys. C 26, 495 (1985).

7. J. Ahrens et al., Eur. Phys. J. A 23, 113 (2005).

8. R. Garcia-Martin, B. Moussallam, hep-ph/1006.5373.

9. L.V. Filkov, V.L. Kashevarev, Phys. Rev. C 81, 029801 (2010).

10. COMPASS Collaboration (P. Abbon et al.), Nucl. Instrum. Methods A 577, 455 (2007).

11. I.Y. Pomeranchuk, I.M. Shmushkevich, Nucl. Phys. 23, 452 (1961).

12. N. Kaiser, J.M. Friedrich, Eur. Phys. J. A 36, 181 (2008).

13. N. Kaiser, J.M. Friedrich, Nucl. Phys. A 812, 186 (2008).

14. N. Kaiser, J.M. Friedrich, Eur. Phys. J. A 39, 71 (2009).

15. J. Gasser, V.E. Lyubovitskij, A. Rusetsky, A. Gall, Phys. Rev. D 64, 016008 (1988).

16. M. Knecht, R. Urech, Nucl. Phys. B 519, 329 (1998).

17. J. Bijnens, F. Cornet, Nucl. Phys. B 296, 557 (1988)

18. J.F. Donoghue, B.R. Holstein, Y.C. Lin, Phys. Rev. D 37, 2423 (1988)

19. S. Bellucci, J. Gasser, M.E. Sainio, Nucl. Phys. B 423, 80 (1994).

20. J.R. Batley et al., Eur. Phys. J. C 64, 589 (2009).

21. N. Cabibbo, G. Isidori, JHEP 0503, 021 (2005).

22. Ulf-G. Meißner, G. Müller, S. Steininger, Phys. Lett. B 406, 154 (1997); 407, 454 (1997)(E). 